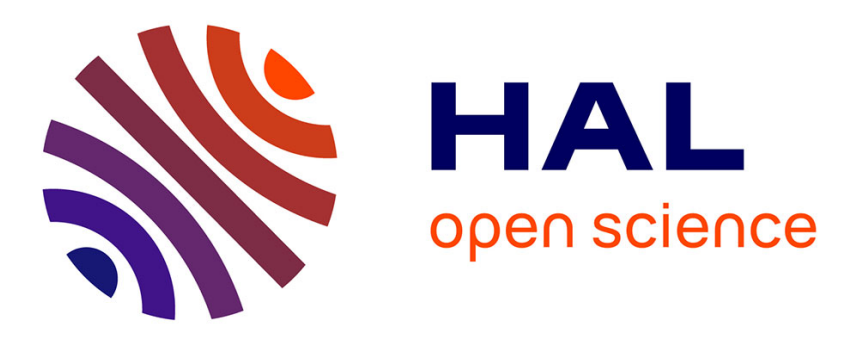

\title{
Detectability of Earth-like planets in circumstellar habitable zones of binary star systems with sun-like components
}

Siegfried Eggl, Nader Haghighipour, Elke Pilat-Lohinger

\section{- To cite this version:}

Siegfried Eggl, Nader Haghighipour, Elke Pilat-Lohinger. Detectability of Earth-like planets in circumstellar habitable zones of binary star systems with sun-like components. The Astrophysical Journal, 2013, 764 (Issue 2), http://iopscience.iop.org/0004-637X/764/2/130. 10.1088/0004-637X/764/2/130 . hal-01078051

\section{HAL Id: hal-01078051 \\ https://hal.science/hal-01078051}

Submitted on 28 Oct 2014

HAL is a multi-disciplinary open access archive for the deposit and dissemination of scientific research documents, whether they are published or not. The documents may come from teaching and research institutions in France or abroad, or from public or private research centers.
L'archive ouverte pluridisciplinaire HAL, est destinée au dépôt et à la diffusion de documents scientifiques de niveau recherche, publiés ou non, émanant des établissements d'enseignement et de recherche français ou étrangers, des laboratoires publics ou privés. 


\title{
DETECTABILITY OF EARTH-LIKE PLANETS IN CIRCUMSTELLAR HABITABLE ZONES OF BINARY STAR SYSTEMS WITH SUN-LIKE COMPONENTS
}

\author{
Siegfried EgGL ${ }^{1,2}$, Nader Haghighipour ${ }^{3}$, and Elke Pilat-Lohinger ${ }^{1}$ \\ ${ }^{1}$ University of Vienna, Institute for Astrophysics, Türkenschanzstr. 17, A-1180 Vienna, Austria; siegfried.eggl@univie.ac.at \\ 2 IMCCE, Observatoir de Paris, 77 Avenue Denfert-Rochereau, F-75014 Paris, France \\ ${ }^{3}$ Institute for Astronomy and NASA Astrobiology Institute, 2680 Woodlawn Drive, Honolulu, HI 96822, USA \\ Received 2012 September 5; accepted 2012 December 19; published 2013 January 31
}

\begin{abstract}
Given the considerable percentage of stars that are members of binaries or stellar multiples in the solar neighborhood, it is expected that many of these binaries host planets, possibly even habitable ones. The discovery of a terrestrial planet in the $\alpha$ Centauri system supports this notion. Due to the potentially strong gravitational interaction that an Earth-like planet may experience in such systems, classical approaches to determining habitable zones (HZ), especially in close S-type binary systems, can be rather inaccurate. Recent progress in this field, however, allows us to identify regions around the star permitting permanent habitability. While the discovery of $\alpha$ Cen Bb has shown that terrestrial planets can be detected in solar-type binary stars using current observational facilities, it remains to be shown whether this is also the case for Earth analogs in HZs. We provide analytical expressions for the maximum and rms values of radial velocity and astrometric signals, as well as transit probabilities of terrestrial planets in such systems, showing that the dynamical interaction of the second star with the planet may indeed facilitate the planets' detection. As an example, we discuss the detectability of additional Earth-like planets in the averaged, extended, and permanent HZs around both stars of the $\alpha$ Centauri system.
\end{abstract}

Key words: astrobiology - celestial mechanics - methods: analytical - planet-star interactions

\section{INTRODUCTION}

The past decades have seen a great number of discoveries of planets around stars other than our Sun (Schneider et al. 2011). As some of these planets are of terrestrial nature, the hope of identifying Earth analogs has lead to considerable advances toward the detection of possibly habitable worlds (Borucki 2011; Ford et al. 2012). Even though quite frequent in the solar neighborhood (Kiseleva-Eggleton \& Eggleton 2001), not many attempts have yet been made to specifically target binary stars in this endeavor. Nonetheless, more than 60 planets have already been found in and around such systems (Haghighipour 2010; Doyle et al. 2011; Welsh et al. 2012; Roell et al. 2012; Orosz et al. 2012a, 2012b; Dumusque et al. 2012). Although several P-type (circumbinary) planets orbiting both stars of a close binary have also been discovered (Doyle et al. 2011; Welsh et al. 2012; Orosz et al. 2012a, 2012b), most planets are in the so-called S-type (Rabl \& Dvorak 1988) configuration where the planet orbits only one of the binary's stars. A prominent example of an S-type system is $\alpha$ Centauri AB which hosts a terrestrial planet around the fainter binary component, $\alpha$ Cen $\mathrm{B}$ (Dumusque et al. 2012).

The reason for the general reluctance to include binary systems in the search for terrestrial, habitable planets lies in the assumption that the additional interactions with a massive companion will make planets harder to find. That is primarily because the gravitational interaction between the second star and a planet may alter the planet's orbit significantly and complicate the task of interpreting the planetary signal. One aim of this work is therefore to show that changes in the planet's orbit can actually enhance its detectability (see Section 5). Of course, the orbit of a binary as well as its stellar parameters have to be well determined in order to be able to identify signals from additional terrestrial planets. Sensing the need for a better understanding of binary star systems, efforts have been intensified to improve physical as well as orbital data for nearby binaries (e.g., Torres et al. 2010) and to evolve existing data analysis methodologies (Chauvin et al. 2011; Haghighipour 2010; Pourbaix 2002; Pourbaix et al. 2002).

Understanding the complex interactions between a stellar binary and a planet is essential if a system's potential habitability is to be evaluated. For instance, one of the main assumptions of classical habitability, as introduced by Kasting et al. (1993), is that the planet moves around its host star on a circular orbit. This may not be a valid assumption for planets in a binary star system where the gravitational perturbation of the secondary can excite the eccentricity of the planet's orbit (Marchal 1990; Georgakarakos 2002; Eggl et al. 2012). Eggl et al. (2012) found that except for S-type systems where the secondary star is much more luminous than the planet's host star, variations in planetary orbit around the planet-hosting star are the main cause for changes in insolation. Even though Eggl et al. (2012) gave an analytic recipe for calculating the boundaries of the habitable zones (HZs) in S-type binaries, it remains to be seen whether an Earth-like planet in the HZ of a system with two Sun-like stars will in fact be detectable.

In order to answer this question, we consider three techniques, namely, radial velocity (RV), astrometry (AM), and transit photometry (TP), and discuss whether the current observational facilities are capable of detecting habitable planets in such systems. We provide analytical formula for estimating the strength of RV and AM signals for habitable, Earth-like planets, and show that the planet-binary interaction can enhance the chances for the detection of these objects.

The rest of this article will be structured as follows. In Sections 2 and 3 analytic estimates of the maximum and root mean square (rms) of the strength of an RV and an AM signal that an Earth-like planet produces in an S-type binary configuration will be derived. Section 4 will deal with the consequences of such a setup for TP. We will then briefly recall the different types of HZs for S-type binaries established in Eggl et al. (2012), and use their methodology to identify similar habitable 
regions in the $\alpha$ Centauri system (Section 5). This system has been chosen because first, it has inspired many studies on the possibility of the formation and detection of habitable planets around its stellar components (Forgan 2012; Guedes et al. 2008; Thébault et al. 2009) and second, Dumusque et al. (2012) have already discovered an Earth-sized planet in a short-period orbit around its secondary star. Therefore, we will compare our RV estimates to the actual signal of $\alpha \mathrm{Cen} \mathrm{Bb}$, and study its influence on an additional terrestrial planet presumed in $\alpha$ Cen B's HZ. Finally, in Section 6, the projected RV, AM, and TP trace that terrestrial planets will leave in the $\mathrm{HZ}$ of the $\alpha$ Centauri system are analyzed, and the results are discussed within the context of the sensitivity of the current observational facilities.

\section{RADIAL VELOCITY}

To estimate the RV signal that an Earth-like planet produces in an S-type binary system, we will build upon the formalism presented by Beaugé et al. (2007). We assume that the nonplanetary contributions to the host star's RV signal (such as the RV variation caused by the motion of the binary around its center of gravity) are known and have been subtracted, leaving behind only the residual signal due to the planet. The motion of the planet around its host star then constitutes a perturbed two-body problem, where the gravitational influence of the secondary star is still playing a role and is mirrored in the forced variations of the planet's orbit.

In practice, the extraction of the planetary signal is all but a trivial task. Even after subtraction of the binary's barycentric and proper motion, the residual will contain contributions from the binary's orbital uncertainties as well as from non-gravitational sources which could be orders of magnitude larger than the star's reflex signal, such as the Rossiter-McLaughlin effect in transiting systems, for example (Ohta et al. 2005). The discovery of $\alpha \mathrm{Cen} \mathrm{Bb}$ showed, however, that a substantial reduction of non-planetary RV interference is possible if the respective binary star has been studied in sufficient detail.

The amplitude of the planet induced RV signal of the host star, $V_{r}$, is given by

$$
V_{r}=K[\cos (f+\omega)+e \cos \omega],
$$

where $K$ is equal to

$$
K=\frac{\mu\left(\kappa n_{p}\right)^{1 / 3} \sin i}{\sqrt{1-e^{2}}} .
$$

In Equation (2), $\mu=m_{1} /\left(m_{0}+m_{1}\right)$ is the planet to star mass ratio with $m_{1}$ and $m_{0}$ being the masses of the planet and host star, respectively. The planet's mean motion, $n_{p}=2 \pi / P_{p}$, is given by $n_{p}=\sqrt{\kappa / a^{3}}$ with $\kappa=\mathcal{G}\left(m_{0}+m_{1}\right)$, and $P_{p}$ and $\mathcal{G}$ being the planet's orbital period and gravitational constant. The quantities $a, e, i, f$, and $\omega$ in Equations (1) and (2) denote the planet's semimajor axis, eccentricity, orbital inclination relative to the plane of the sky, true anomaly, and argument of periastron, respectively.

Our goal in this section is to identify the range of the possible peak amplitudes that a terrestrial planet in an S-type binary configuration can produce. We note that the gravitational influence of the second star causes the planet's orbital elements to vary, thus inducing additional time-dependent changes in the RV signal $V_{r}$ (Lee \& Peale 2003). While we know from secular perturbation theory that $a$ does not change significantly with
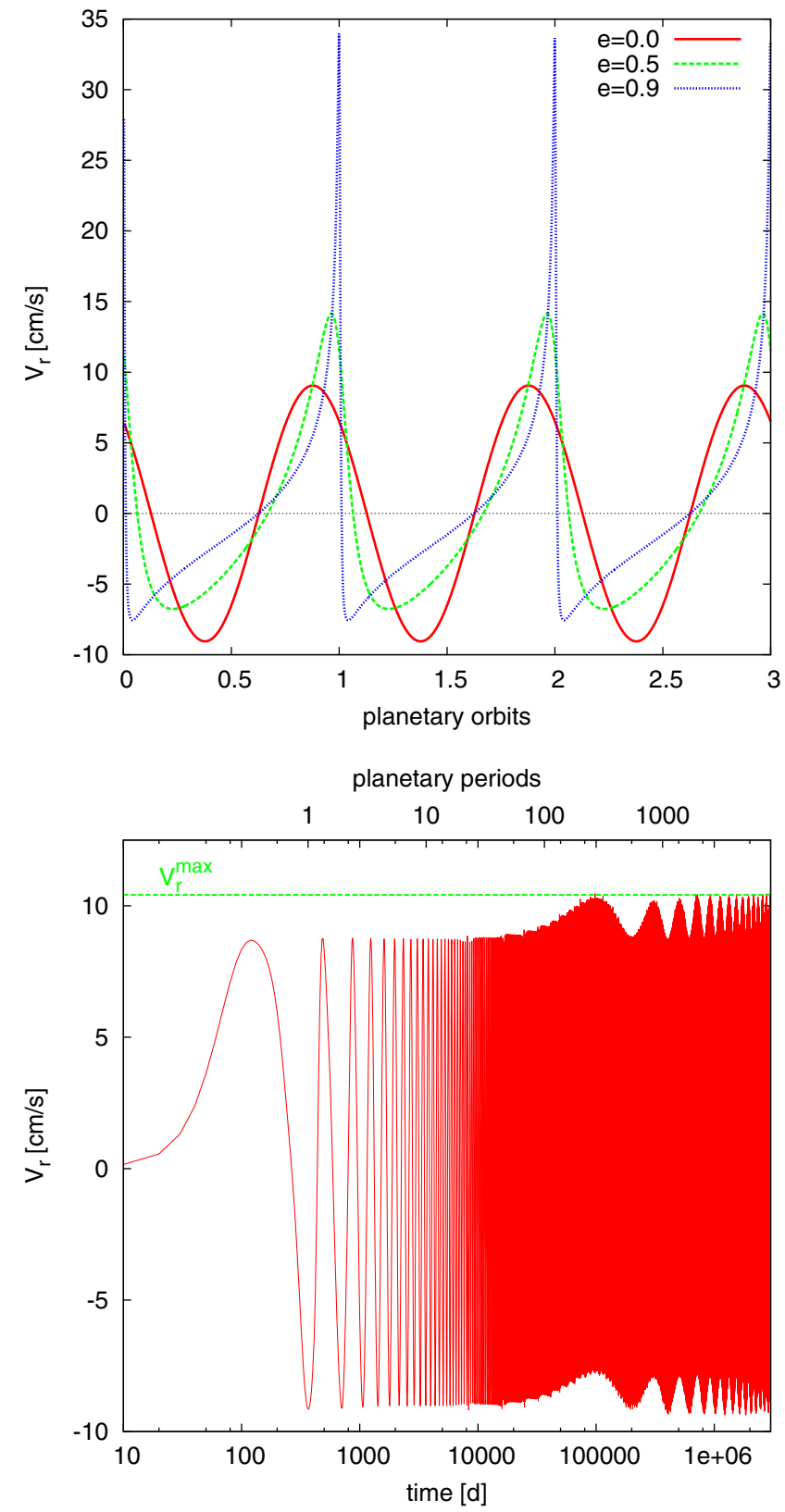

Figure 1. Top: the radial velocity signal caused by an Earth-like planet orbiting a Sun-like star with different eccentricities (Beaugé et al. 2007). The planet is at $1 \mathrm{AU}$ with $\omega=45^{\circ}$ when $e \neq 0$. Bottom: the amplitude variations of the primary's radial velocity signal due to an Earth-like planet that is subject to the gravitational perturbations of a second star. Both stars are Sun-like with a separation of $20 \mathrm{AU}$ and an orbital eccentricity of 0.5 . The planet's initial orbit was circular with a semimajor axis of 1 AU. Our analytically estimated maximum amplitude $V_{r}^{\max }$ is also shown.

(A color version of this figure is available in the online journal.)

time for hierarchical systems such as the one under consideration (Marchal 1990; Georgakarakos 2003), $\omega$ becomes a function of time. We assume coplanar orbits of the planet and the binary star which result in the planet's inclination to the plane of the sky (i) to remain constant. In contrast, the planetary eccentricity will vary between zero and a maximum $e^{\max }$, where the latter value can be expressed as a function of the system's masses and the binary's orbital parameters (Eggl et al. 2012). This is important, because the reflex RV signal $\left(V_{r}\right)$ of a star can be increased significantly by planetary orbital eccentricities (Figure 1). Using Equation (1), we identify the global maximum 
of $V_{r}$ at $f=\omega=0$, when $e=e^{\max }$. This leads to

$$
V_{r}^{\max }=V_{r}^{\text {circ }} \sqrt{\frac{1+e^{\max }}{1-e^{\max }}}
$$

where

$$
V_{r}^{\mathrm{circ}}=\frac{\sqrt{\mathcal{G}} m_{1} \sin i}{\sqrt{a\left(m_{0}+m_{1}\right)}} .
$$

Equation (3) presents a fully analytic estimate of the expected maximum RV signal that a terrestrial planet produces in an S-type binary configuration. ${ }^{4}$

As an example for the influence of a double star on a planetary $\mathrm{RV}$ signal, the induced variations in the RV of the planet's host star are presented in the bottom panel of Figure 1. The host star is a constituent of a solar-type binary with a semimajor axis of 20 $\mathrm{AU}$ and an orbital eccentricity of 0.5. Changes in the amplitude of $V_{r}$ are due to variations in the planet's eccentricity.

Since we do not know the state of the planet's orbital eccentricity at the time of observation, we consider a range for the maximum possible amplitudes of its RV

$$
V_{r}^{\text {circ }} \leqslant\left. V_{r}\right|_{f=\omega=0} \leqslant V_{r}^{\max } .
$$

Although the range of the amplitude of the host star's RV signal, as given by Equation (5), can be used to identify the "best case" detectability limits, the maximum values of the RV signal due to the planet will be "snapshots" that are reached only during brief moments. As a result, their values for assessing the precision needed to trace fingerprints of an Earth-like planet are rather limited. In such cases, expressions for the rms of the astrometric signal are preferable.

Since rms values are by convention time-averaged, we substitute $f$ by the mean anomaly $M=n_{p} t$ in all corresponding functions using the equation of the center expansion up to the sixth order in planetary eccentricities (see Appendix A) and average over $M$ and $\omega$. The vastly different rates of change of these quantities $(\dot{M} \gg \dot{\omega})$ make it possible to consider $\omega$ to remain constant during one cycle of $M$, so that independent averaging can be performed. In order to eliminate short-term variations in the RV signal, we first average over $M$. Averaging over $\omega$ as well might be desirable if for example the initial state of $\omega$ is unknown, or if observations stretch beyond secular evolution timescales of the planets argument of pericenter. We, therefore, define two different types of rms evaluations for a square-integrable function $F$ :

$$
\langle\langle F\rangle\rangle_{M}=\left\langle F^{2}\right\rangle_{M}^{1 / 2}=\left[\frac{1}{2 \pi} \int_{0}^{2 \pi} F^{2}(M) d M\right]^{1 / 2},
$$

and

$$
\langle\langle F\rangle\rangle_{M, \omega}=\frac{1}{2 \pi}\left[\iint_{0}^{2 \pi} F^{2}(M, \omega) d M d \omega\right]^{1 / 2} .
$$

Using Equations (6) and (7), the rms values of $V_{r}$ are then given by

\footnotetext{
4 Larger signals are possible, if the terrestrial planet has a considerable initial eccentricity after its formation and migration phase. Yet, due to the eccentricity dampening in protoplanetary disks, this seems unlikely (Paardekooper \& Leinhardt 2010).
}

$$
\begin{aligned}
\left\langle\left\langle V_{r}\right\rangle\right\rangle_{M}= & \left\langle\left\langle V_{r}\right\rangle\right\rangle_{M, \omega} \\
& \times\left\{1-\left[\frac{\left\langle e^{2}\right\rangle_{M}}{4}+O\left(\left\langle e^{2}\right\rangle_{M}^{2}\right)\right] \cos (2 \omega)\right\}^{1 / 2},
\end{aligned}
$$

with

$$
\left\langle\left\langle V_{r}\right\rangle\right\rangle_{M, \omega}=\frac{\sqrt{\mathcal{G}} m_{1}|\sin i|}{\sqrt{2 a\left(m_{0}+m_{1}\right)}}=\frac{1}{\sqrt{2}} V_{r}^{\text {circ }} .
$$

Here we have considered $\langle a\rangle_{M}=a$ since $\dot{a} \simeq 0$ (Marchal 1990; Georgakarakos 2003). Also

$$
\int_{0}^{2 \pi}\left\langle e^{2}\right\rangle_{M} \cos (2 \omega) d \omega=0
$$

as indicated in Appendix B. It is noteworthy that the averaging over $\omega$ causes the rms value of the RV signal to become independent of $e$ so that its difference with the peak signal in the circular case $\left(V_{r}^{\text {circ }}\right)$ becomes a mere factor of $1 / \sqrt{2}$. Thanks to their intricate relation to power spectra, rms values can also be valuable for orbit fitting. The choice of singly or doubly averaged rms relations for this purpose will depend on how many planetary orbital periods are available in the data set. In the case of $\alpha \mathrm{Cen} \mathrm{Bb}$, there are order-of-magnitude differences in the rates of change of the mean anomaly $(M)$ and the argument of pericenter $(\dot{\omega})$. It would therefore make more sense to assume $\omega$ to be constant and add it as a variable in the fitting process. If stronger perturbations or additional forces act on the planet, the periods can be considerably shorter, so that the fully averaged equations might come in handy.

\section{ASTROMETRY}

In order to derive the maximum and rms values for an astrometric signal, we will use the framework presented in Pourbaix (2002). We again assume that the non-planetary contributions have been subtracted from the combined signal of the host star and planet. The projected motion of the planet on the astrometric plane is then given by

$$
\begin{aligned}
& x_{E}=A(\cos E-e)+F \sqrt{1-e^{2}} \sin E, \\
& y_{E}=B(\cos E-e)+G \sqrt{1-e^{2}} \sin E,
\end{aligned}
$$

where $x_{E}$ and $y_{E}$ are the Cartesian coordinates of the projected orbit, $e$ is the planet's orbital eccentricity, $E$ is the eccentric anomaly, and $A, B, F$, and $G$ are the modified Thiele-Innes constants given by

$$
\begin{aligned}
A & =\frac{a}{d}(\cos \omega \cos \Omega-\sin \omega \sin \Omega \cos i), \\
B & =\frac{a}{d}(\cos \omega \sin \Omega+\sin \omega \cos \Omega \cos i), \\
F & =-\frac{a}{d}(\sin \omega \cos \Omega+\cos \omega \sin \Omega \cos i), \\
G & =-\frac{a}{d}(\sin \omega \sin \Omega-\cos \omega \cos \Omega \cos i) .
\end{aligned}
$$

In these equations, $d$ is the distance between the observer and the observed system in units of the planetary semimajor axis $a$. We can rewrite Equations (10) in terms of the true anomaly $f$ as

$$
\begin{aligned}
& x_{f}=\frac{A}{a} r \cos f+\frac{F}{a} r \sin f, \\
& y_{f}=\frac{B}{a} r \cos f+\frac{G}{a} r \sin f .
\end{aligned}
$$


In these equations, $r=a\left(1-e^{2}\right) /(1+e \cos f)$ represents the planet's radial distance to its host star. Because the motion of the planet itself cannot be traced, we translate these equations into the apparent motion of the host star by the application of Newton's third law. That is

$$
\begin{aligned}
& x_{\star}=X-\mu x_{f}, \\
& y_{\star}=Y-\mu y_{f} .
\end{aligned}
$$

Here, $X$ and $Y$ are the projected coordinates of the center of mass of the planet-star system, and $\mu$ denotes the planet-star mass ratio as defined for Equation (2).

Assuming without the loss of generality that the barycenter of the star-planet system coincides with the origin of the associated coordinate system, the distance of the projected stellar orbit to the coordinate center will be equal to

$$
\begin{aligned}
\rho^{2} & =x_{\star}^{2}+y_{\star}^{2} \\
& =\frac{\mu^{2} a^{2}}{d^{2}} \frac{\left(1-e^{2}\right)^{2}\left[1-\sin ^{2} i \sin ^{2}(f+\omega)\right]}{(1+e \cos f)^{2}} .
\end{aligned}
$$

The right-hand side of Equation (14) is independent of $\Omega$ and has a global maximum at $f=\pi, \omega=0$ when $e=e^{\max }$. This translates into a maximum astrometric amplitude given by

$$
\rho^{\max }=\rho^{\operatorname{circ}}\left(1+e^{\max }\right),
$$

where

$$
\rho^{\mathrm{circ}}=\frac{\mu a}{d} .
$$

The planetary maximum AM signal will again lie between $\rho^{\text {circ }}$ and $\rho^{\max }$. A remarkable feature of $\rho^{\max }$ and $\rho^{\text {circ }}$ is their independence of the system's inclination $i$. This is visualized in Figure 2. The same figure also shows the time evolution of the AM signal due to an Earth-like planet orbiting $\alpha$ Cen B at a distance of 1 AU.

The astrometric rms values are given by

$$
\begin{aligned}
\langle\langle\rho\rangle\rangle_{M}= & \rho^{\operatorname{circ}}\left[1+\frac{3\left\langle e^{2}\right\rangle_{M}}{2}\right. \\
& \left.+\left(-\frac{1}{2}+\frac{\left\langle e^{2}\right\rangle_{M}}{4}(5 \cos [2 \omega]-3)\right) \sin ^{2} i\right]^{1 / 2}
\end{aligned}
$$

and

$$
\begin{aligned}
\langle\langle\rho\rangle\rangle_{M, \omega}= & \frac{\rho^{\mathrm{circ}}}{2}\left[3+\frac{9}{2}\left\langle e^{2}\right\rangle_{M, \omega}\right. \\
& \left.+\left(1+\frac{3}{2}\left\langle e^{2}\right\rangle_{M, \omega}\right) \cos (2 i)\right]^{1 / 2} .
\end{aligned}
$$

Details regarding the derivation of Equations (17) and (18) can be found in Appendix B. In contrast to the doubly averaged equations for the rms of an RV signal, Equation (18) shows a dependence on the binary's eccentricity. In cases where the planetary inclination $i$ coincides with the inclination of the binary itself, analytic expressions for $\left\langle e^{2}\right\rangle_{M, \omega}$ are available (Georgakarakos 2003, 2005). ${ }^{5}$

\footnotetext{
5 The analytic expressions given in these articles are also averaged over initial phases, i.e., different relative starting positions of the planet and the binary stars.
}
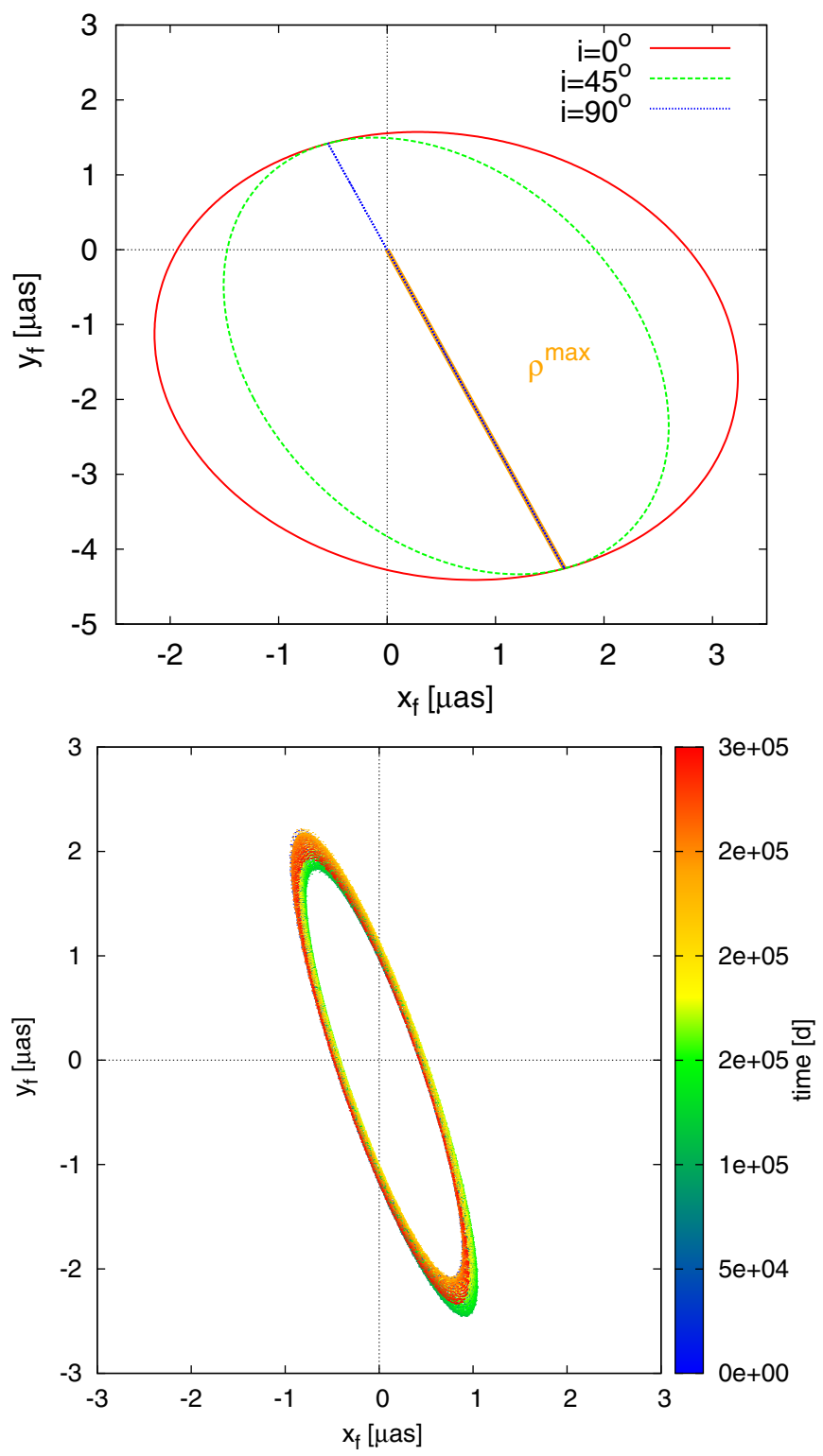

Figure 2. Top: the maximum astrometric amplitude, $\rho^{\max }=$ $\left.\mu\left(x_{f}^{2}+y_{f}^{2}\right)^{1 / 2}\right|_{f=\pi, \omega=0}$, due to an Earth-like planet orbiting its Sunlike host star. The planet's orbital elements are $a=1 \mathrm{AU}, e=e^{\max }=0.5$, $\omega=0, \Omega=111^{\circ}$. As shown here, the maximum distance from the origin of the coordinate system is independent of the system's inclination with respect to the plane of the sky $(i)$. Bottom: evolution of the astrometric signal $\left(x_{f}, y_{f}\right)$ caused by an Earth-like planet in a binary star system. The planet is orbiting $\alpha$ Cen $\mathrm{B}$ at a distance of $1 \mathrm{AU}$. The evolution of the astrometric signal is shown for 3750 periods of $\alpha$ Centauri AB. Since the system is coplanar, the changes in orientation and shape of the projected ellipse are due to variations in the planet's eccentricity $(e)$ and argument of pericenter $(\omega)$.

\section{TRANSIT PHOTOMETRY}

In TP, signal strength is equivalent to the relative depth of the dint the planet produces in the stellar light curve during its transit. Assuming that the star-planet configuration allows for occultations, and excluding grazing transits, the fractional depth of the photometric transit (TD) produced by an Earth-like planet is simply given by the proportion of the luminous area of the disk of the star that is covered by the planet as the planet moves between the observer and the star. Ignoring limb darkening, that means, $\mathrm{TD} \simeq R_{p}^{2} / R_{\star}^{2}$ where $R_{p}$ is the radius of the planet and $R_{\star}$ is the stellar radius. The overall probability to observe a transit 
Table 1

Physical and Orbital Parameters of the $\alpha$ Centauri AB $b$ System (Kervella et al. 2003; Guedes et al. 2008; Pourbaix et al. 2002; Dumusque et al. 2012)

\begin{tabular}{lcc}
\hline \hline$\alpha$ Centauri & $\mathrm{A}$ & $\mathrm{B}$ \\
\hline Spectral classification & $\mathrm{G} 2 \mathrm{~V}$ & $\mathrm{~K} 1 \mathrm{~V}$ \\
Mass $\left(M_{\odot}\right)$ & $1.105 \pm 0.007$ & $0.934 \pm 0.007$ \\
$T_{\text {eff }}(\mathrm{K})$ & 5790 & 5260 \\
Luminosity $\left(L_{\odot}\right)$ & 1.519 & 0.500 \\
\hline Distance $(\mathrm{pc})$ & $1.339 \pm 0.002$ \\
Period $\left(P_{b}\right)($ days $)$ & $29187 \pm 4$ \\
$a_{b}(\mathrm{AU})$ & $23.4 \pm 0.03$ \\
$e_{b}$ & $0.5179 \pm 0.00076$ \\
$i_{b}($ deg $)$ & $79.205 \pm 0.0041$ \\
$\omega_{b}(\mathrm{deg})$ & $231.65 \pm 0.076$ \\
$\Omega_{b}(\mathrm{deg})$ & $204.85 \pm 0.084$ \\
\hline$\alpha$ Centauri & \multicolumn{2}{c}{$\mathrm{B} \mathrm{b}$} \\
\hline$P_{p}($ days $)$ & \multicolumn{2}{c}{$3.2357 \pm 0.0008$} \\
$e_{p}$ & 0 (fixed $)$ \\
Minimum mass $\left(m_{p}^{\min }\right)\left(M_{\text {Earth }}\right)$ & $1.13 \pm 0.09$ \\
\hline
\end{tabular}

is given by (Borucki \& Summers 1984)

$$
p_{T}=\frac{R_{\star}}{r_{T}} .
$$

In Equation (19), $r_{T}$ is the radial distance of the planet to the star during the transit. For an eccentric planetary motion, the planet-star distance during transit can be expressed as $r_{T} \simeq a\left(1-e^{2}\right) /(1+e \cos \bar{\omega})$ (Ford et al. 2008), where $\bar{\omega}$ denotes the argument of pericenter measured from the line of sight. ${ }^{6}$ In analogy to Sections 2 and 3, the maximum and averaged transit probability for a planet perturbed by the secondary star in a planar configuration can be calculated by substituting for $r_{T}$ in Equation (19) and averaging over $\bar{\omega}$. This will result in

$$
p_{T}^{\max } \simeq \frac{R_{\star}}{a\left(1-e^{\max }\right)},
$$

and

$$
\left\langle p_{T}\right\rangle_{\bar{\omega}} \simeq \frac{R_{\star}}{a\left(1-\left\langle e^{2}\right\rangle_{M, \omega}\right)} .
$$

Equations (20) and (21) indicate that the increase in the eccentricity of the planet due to the perturbation of the secondary increases the probability of transit. In deriving these equations, we have ignored the occultation of the planet by the second star. However, depending on the period ratio between the secondary and the planet, such conjunctions are either scarce or short-lived. Consequently, their contribution to the probability of witnessing a planetary transit is negligibly small.

\section{APPLICATION TO THE $\alpha$ CENTAURI SYSTEM}

In this section, we will show that the previously derived analytic expressions produce results that are in good agreement with the current observations of $\alpha \mathrm{Cen} \mathrm{Bb}$. We will also present numerical evidence that the presence-or the absence-of an additional terrestrial planet in the $\mathrm{HZ}$ of $\alpha$ Cen $\mathrm{B}$ cannot be derived easily from the orbit evolution of $\alpha \mathrm{Cen} \mathrm{Bb}$. Consequently, we argue that an independent detection of additional

\footnotetext{
6 Note that this is different from the conventions used for RV and AM measurements.
}

Table 2

A Comparison between the Predicted RV Values Using the Analytic Expressions Derived in Section 2 and the Observed Values for the Terrestrial Planet Discovered around $\alpha$ Cen B (Dumusque et al. 2012).

\begin{tabular}{lcc}
\hline \hline & $\begin{array}{c}\text { Predicted Signal } \\
\left(\mathrm{m} \mathrm{s}^{-1}\right)\end{array}$ & $\begin{array}{c}\text { Observed Signal } \\
\left(\mathrm{m} \mathrm{s}^{-1}\right)\end{array}$ \\
\hline$\left\langle\left\langle V_{r}\right\rangle\right\rangle_{M, \omega}$ & $0.365 \pm 0.029$ & \\
$V_{r}^{\text {circ }}$ & $0.517 \pm 0.041$ & $0.51 \pm 0.04$ \\
$V_{r}^{\max }$ & $0.519 \pm 0.041$ & \\
\hline
\end{tabular}

Notes. Coplanarity of the system was assumed. The formal uncertainties have been derived assuming Gaussian error propagation of the uncertainties given in Table 1. The maximum predicted planetary eccentricity for $\alpha$ Cen $\mathrm{B} b$ is $e^{\max }=0.003$. When taking general relativity into account, however, the orbit of $\alpha$ Cen $\mathrm{B} b$ will remain practically circular (see Figure 6).

terrestrial companions might be difficult, but more promising. For this purpose, we will determine the $\mathrm{HZ}$ of $\alpha$ Cen B, as well as the RV, AM, and TP signatures of an Earth-like planet orbiting in the HZ of $\alpha$ Cen B. Since there is no a priori reason why the brighter component of $\alpha$ Centauri could not be hosting a terrestrial planet as well, we perform a similar analysis for $\alpha$ Cen A. We will also study the behavior of Equations (1)-(21) for a broad range of binary eccentricities.

\section{1. $\alpha$ Centauri's Terrestrial Planet}

The planet discovered around $\alpha$ Cen B offers a perfect opportunity to compare the RV amplitude predictions derived in Section 2 with actual measurements. The planet's known orbital parameters are given in Table 1 . In Table 2, we present the analytic estimates of Section 2 applied to the $\alpha$ Centauri ABb system. Assuming the system to be coplanar $\left(i \simeq 79^{\circ} .2\right)$, the predicted RV amplitude for circular planetary motion $\left(V_{r}^{\text {circ }}\right)$ is very close to the observed RV amplitude. This is not surprising, since the planetary parameters were derived from an RV signal using the same methodology in reverse. While still well within measurement uncertainties, the deviation of the maximum RV amplitude $\left(V_{r}^{\max }\right)$ from the observed value is larger than that of $V_{r}^{\text {circ }}$. On the one hand, this might indicate that the planet is currently in an orbital evolution phase where its eccentricity is almost zero. On the other hand, the planet may be too close to its host star for our model to predict $V_{r}^{\max }$ correctly. In fact, we show in Section 5.3 that the latter explanation is more likely, since the influence of general relativity (GR) cannot be neglected in this case. Estimates based on Newtonian physics exaggerate the actual eccentricity of $\alpha \mathrm{Cen} \mathrm{Bb}$. Its orbit remains practically circular despite the interaction with the binary star (see Section 5.3 for a detailed discussion). This justifies the assumption of a circular planetary orbit made by Dumusque et al. (2012).

Since we are especially interested in additional habitable planets, however, it is worthwhile to ask whether predictions on the orbital evolution of $\alpha \mathrm{Cen} \mathrm{Bb}$ can be used to exclude the presence of other gravitationally active bodies in the system. In other words, could an Earth-like planet still orbit in the HZ of $\alpha$ Cen B or would the accompanying distortions of the orbit of $\alpha$ Cen $\mathrm{Bb}$ be significant enough to detect them immediately? Before we try to answer these questions, we need to briefly recall some important aspects regarding HZs in binary star systems.

\subsection{Classification of $H Z s$}

Combining the classical definition of an HZ (Kasting et al. 1993) with the dynamical properties of a planet-hosting double 
$\alpha$ Cen A
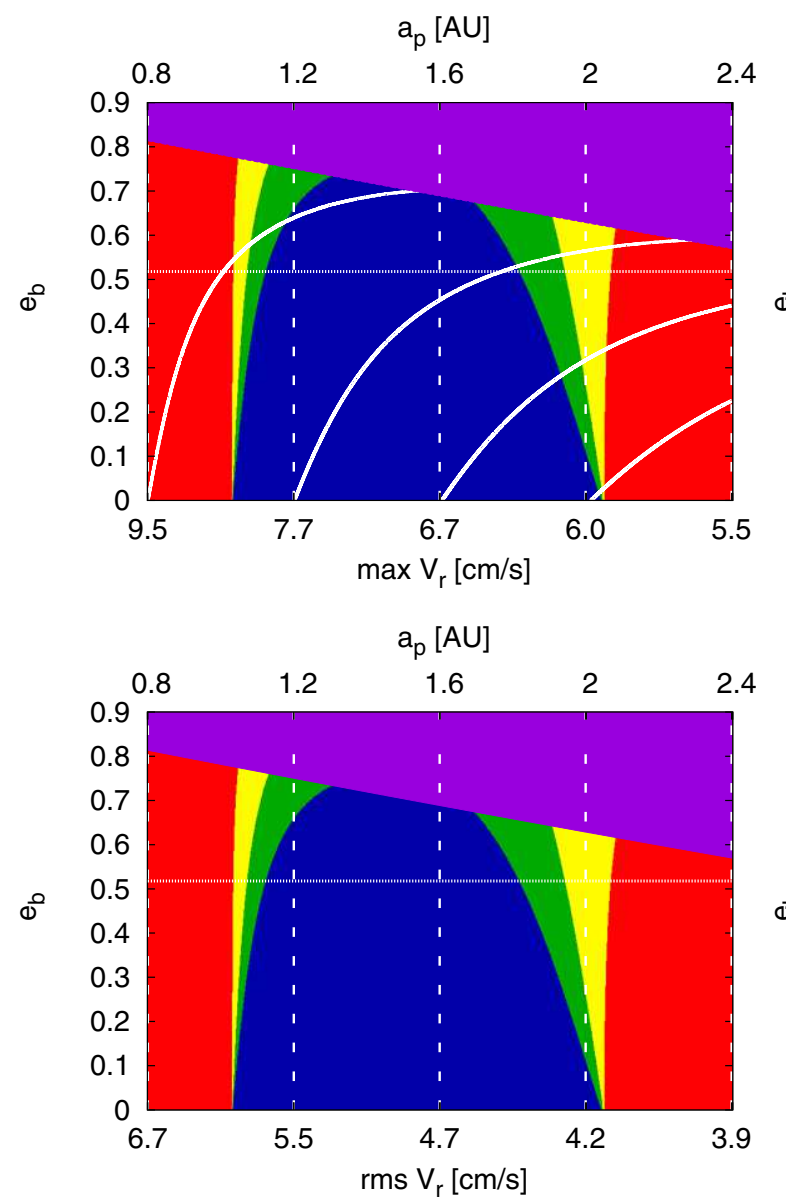

$\alpha$ Cen B
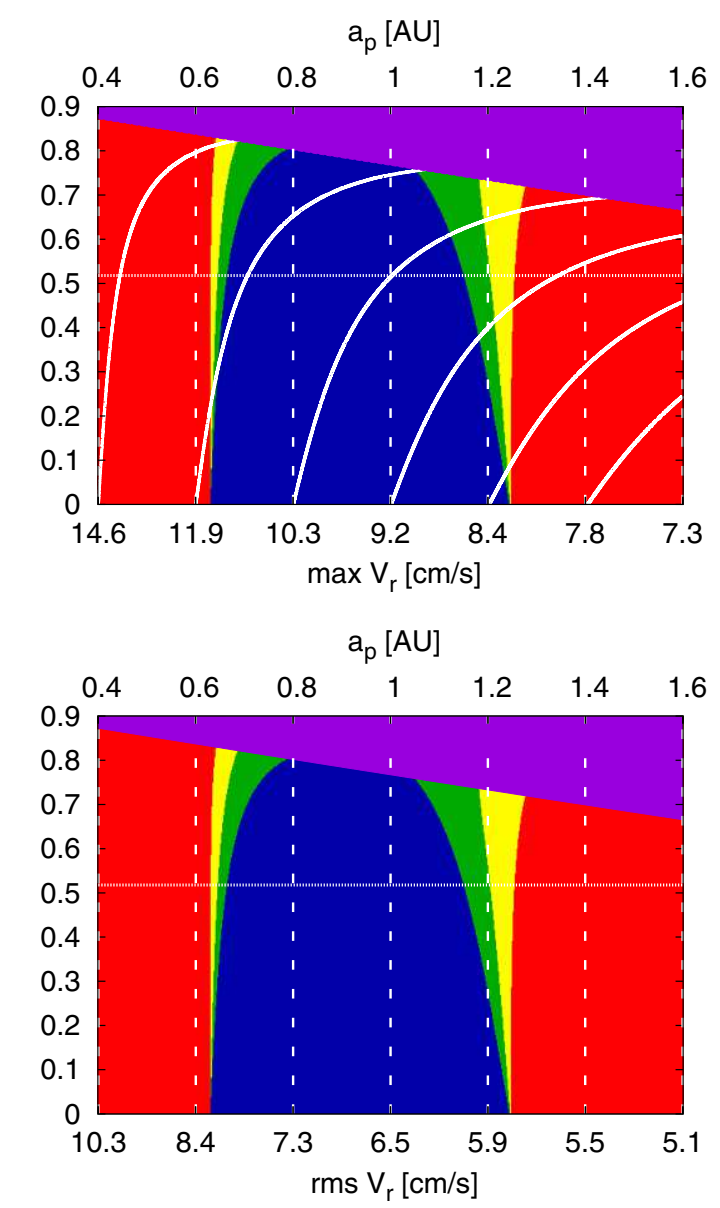

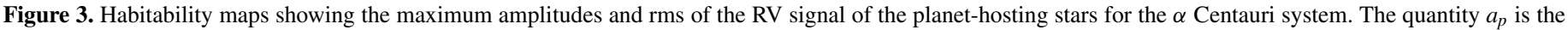

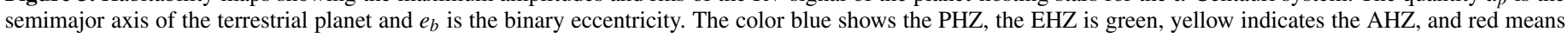

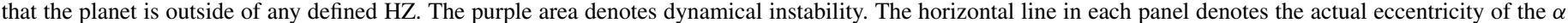

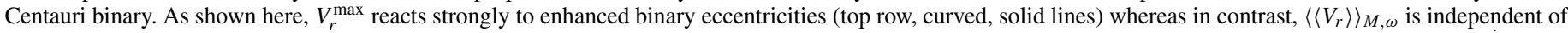

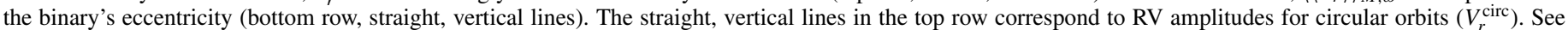
Section 5.4 for details.

star system, Eggl et al. (2012) have shown that one can distinguish three types of HZ in an S-type binary system.

The permanently habitable zone (PHZ) where a planet always stays within the insolation limits $\left(S_{I}, S_{O}\right)$ as defined by Kasting et al. (1993) and Underwood et al. (2003). In other words, despite the changes in its orbit, the planet never leaves the classical HZ. The total insolation the planet receives will vary between the inner $\left(S_{I}\right)$ and outer $\left(S_{O}\right)$ effective radiation limits as $S_{I} \geqslant S_{\text {tot }} \geqslant S_{O}$ where, for a given stellar spectral type, $S_{I}$ and $S_{O}$ are in units of solar constant $\left(1360 \mathrm{~W} \mathrm{~m}^{-2}\right)$.

The extended habitable zone (EHZ) where, in contrast to the PHZ, parts of the planetary orbit may lie outside the HZ due to the planet's high eccentricity, for instance. Yet, the binary-planet configuration is still considered to be habitable when most of the planet's orbit remains inside the boundaries of the HZ. In this case, $\left\langle S_{\text {tot }}\right\rangle_{t}+\sigma \leqslant S_{I}$ and $\left\langle S_{\text {tot }}\right\rangle_{t}-\sigma \geqslant S_{O}$ where $\left\langle S_{\text {tot }}\right\rangle_{t}$ denotes the time-averaged effective insolation from both stars and $\sigma^{2}$ is the effective insolation variance.

The averaged habitable zone (AHZ). Following the argument of Williams \& Pollard (2002) that planetary eccentricities up to $e<0.7$ may not be prohibitive for hab- itability as long as the atmosphere can act as a buffer, the AHZ is defined as encompassing all configurations which support the planet's time-averaged effective insolation to be within the limits of the classical HZ. Therefore, $S_{I} \geqslant\left\langle S_{\text {tot }}\right\rangle_{t} \geqslant S_{O}$.

Analytic expressions for the maximum insolation, the average insolation $\left(\left\langle S_{\text {tot }}\right\rangle\right)$, and insolation variance that a planet encounters in a binary system have been derived in Eggl et al. (2012). We refer the reader to that article for more details.

Figures 3 and 4 show the application of the proposed habitability classification scheme to the $\alpha$ Centauri system. In these figures, blue denotes PHZs, green shows EHZs, and yellow corresponds to AHZs. The red areas in Figures 3 and 4 are uninhabitable, and purple stands for dynamically unstable regions. Table 1 shows the physical properties of the system. We used the formulae by Underwood et al. (2003) to calculate $S_{I}$ and $S_{O}$ for the given effective temperatures of $\alpha$ Cen A and B. In general, these formulae allow for extending the analytic estimates for HZs, as given by Eggl et al. (2012), to main-sequence stars with different spectral types. Runaway greenhouse and maximum greenhouse insolation limits were used to determine the inner and outer boundaries of HZs, respectively. 

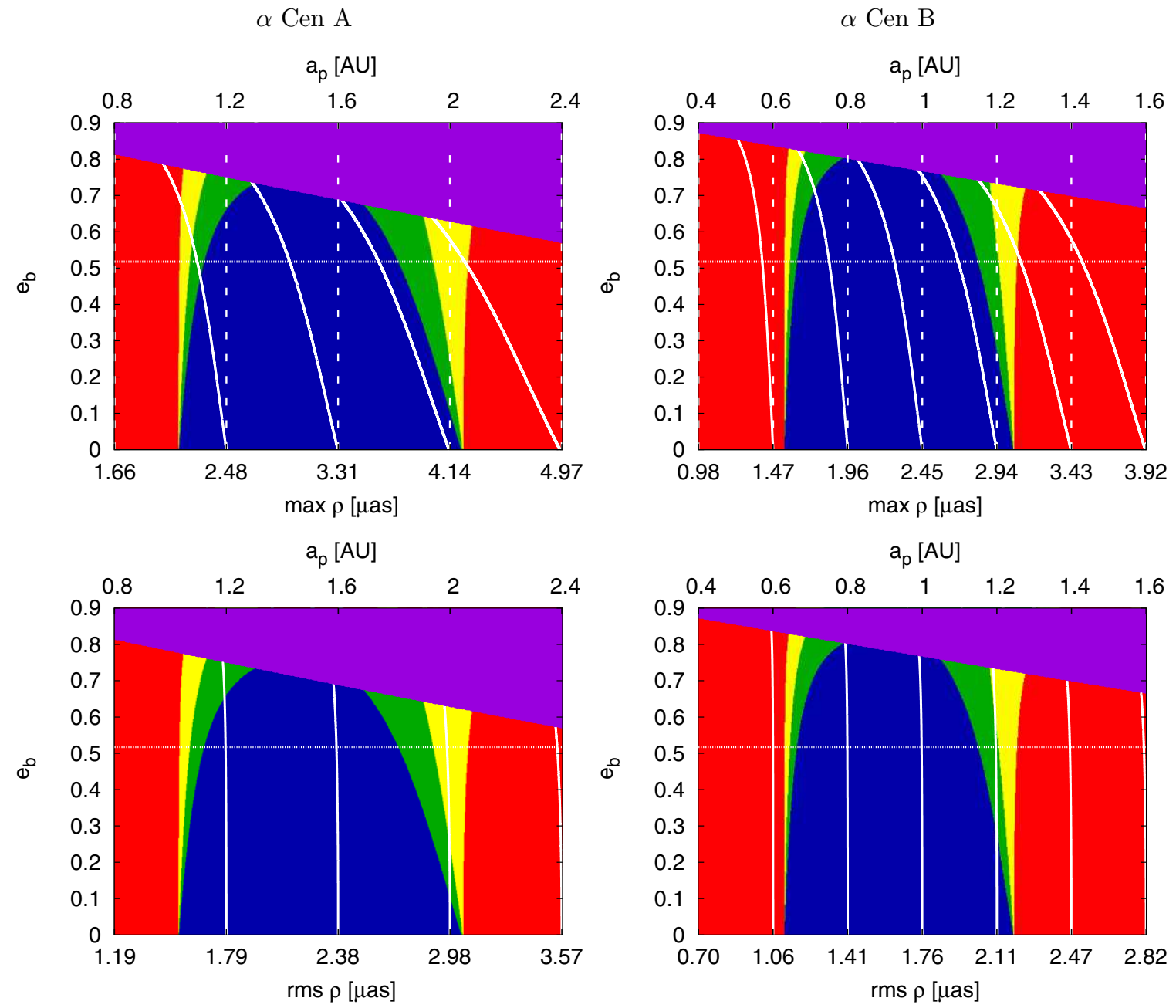

Figure 4. Habitability maps showing the maximum amplitudes and rms of the astrometric signals for the $\alpha$ Centauri system. The color coding is similar to Figure 3 . The vertical dashed lines in the top panels represent regions with similar values of $\rho^{\text {circ }}$. The curved lines in these panels show regions with similar $\rho^{\text {max }}$ amplitudes. In the bottom panels, the vertical lines represent areas of equal rms amplitudes, $\left\langle\langle\rho\rangle_{M, \omega}\right.$. One can see that planetary orbits with dynamically enhanced eccentricities can have smaller semimajor axes and still produce similarly high astrometric amplitudes as circular orbits which are more distant from the host star.

Table 3

Detectability of an Earth-like Planet in the HZs of the $\alpha$ Centauri System.

\begin{tabular}{|c|c|c|c|c|c|c|c|c|c|}
\hline$\alpha$ Cen & $\begin{array}{c}a_{c} \\
(\mathrm{AU})\end{array}$ & Inner AHZ & Inner EHZ & Inner PHZ & Outer PHZ & Outer EHZ & Outer AHZ & & \\
\hline \multirow{5}{*}{ A } & \multirow{5}{*}{2.76} & 1.03 & 1.07 & 1.12 & 1.81 & 1.94 & 2.06 & HZ border & $(\mathrm{AU})$ \\
\hline & & 8.97 & 8.83 & 8.66 & 7.14 & 6.97 & 6.82 & $V_{r}^{\max }$ & \multirow{2}{*}{$\left(\mathrm{cm} \mathrm{s}^{-1}\right)$} \\
\hline & & 5.89 & 5.78 & 5.65 & 4.44 & 4.30 & 4.17 & $\left\langle\left\langle V_{r}\right\rangle\right\rangle_{M, \omega}$ & \\
\hline & & 2.28 & 2.37 & 2.49 & 4.20 & 4.52 & 4.84 & $\rho^{\max }$ & \multirow{2}{*}{ ( $\mu$ as $)$} \\
\hline & & 1.53 & 1.59 & 1.66 & 2.69 & 2.88 & 3.06 & $\langle\langle\rho\rangle\rangle_{M, \omega}$ & \\
\hline \multirow{5}{*}{ B } & \multirow{5}{*}{2.51} & 0.62 & 0.64 & 0.65 & 1.13 & 1.19 & 1.23 & HZ border & $(\mathrm{AU})$ \\
\hline & & 12.21 & 12.09 & 11.94 & 9.37 & 9.19 & 9.04 & $V_{r}^{\max }$ & \multirow{2}{*}{$\left(\mathrm{cm} \mathrm{s}^{-1}\right)$} \\
\hline & & 8.25 & 8.16 & 8.05 & 6.12 & 5.98 & 5.86 & $\left\langle\left\langle V_{r}\right\rangle\right\rangle_{M, \omega}$ & \\
\hline & & 1.58 & 1.62 & 1.66 & 2.97 & 3.12 & 3.26 & $\rho^{\max }$ & \multirow{2}{*}{ ( $\mu$ as) } \\
\hline & & 1.09 & 1.11 & 1.14 & 1.98 & 2.08 & 2.16 & $\langle\langle\rho\rangle\rangle_{M, \omega}$ & \\
\hline
\end{tabular}

Notes. Each row shows the maximum amplitude of the radial velocity signal as well as the astrometric fingerprints of a terrestrial planet in the $\alpha$ Centauri HZs. The critical planetary semimajor axis $\left(a_{c}\right)$ indicates the onset of dynamical instability (Holman \& Wiegert 1999). Computations using chaos indicators are in good agreement with those stability limits (Pilat-Lohinger \& Dvorak 2002). Analytic expressions for calculating the boundary values of planetary semimajor axes in the system's HZs are given in Eggl et al. (2012).

As shown in Figures 3 and 4, the locations of the HZs and the detectability of habitable planets in those regions depend strongly on the eccentricity of the binary $\left(e_{b}\right)$. The actual eccentricity of the $\alpha$ Centauri system is denoted by a horizontal line at $e_{b}=0.5179$. The values for the borders of the different HZs using $\alpha$ Centauri's actual eccentricity are listed in Table 3. As shown here, both stars permit dynamical stability for habitable, Earth-like planets. Due to the difference 


$$
\alpha \text { Cen A }
$$
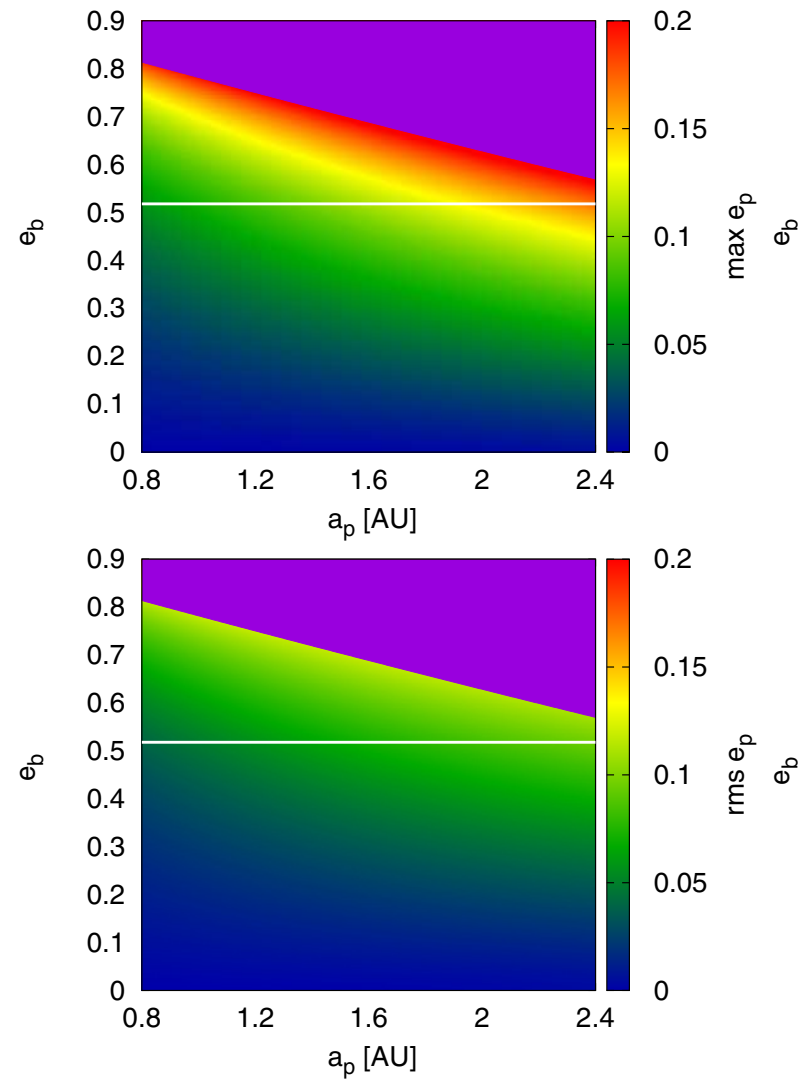

$\alpha$ Cen B
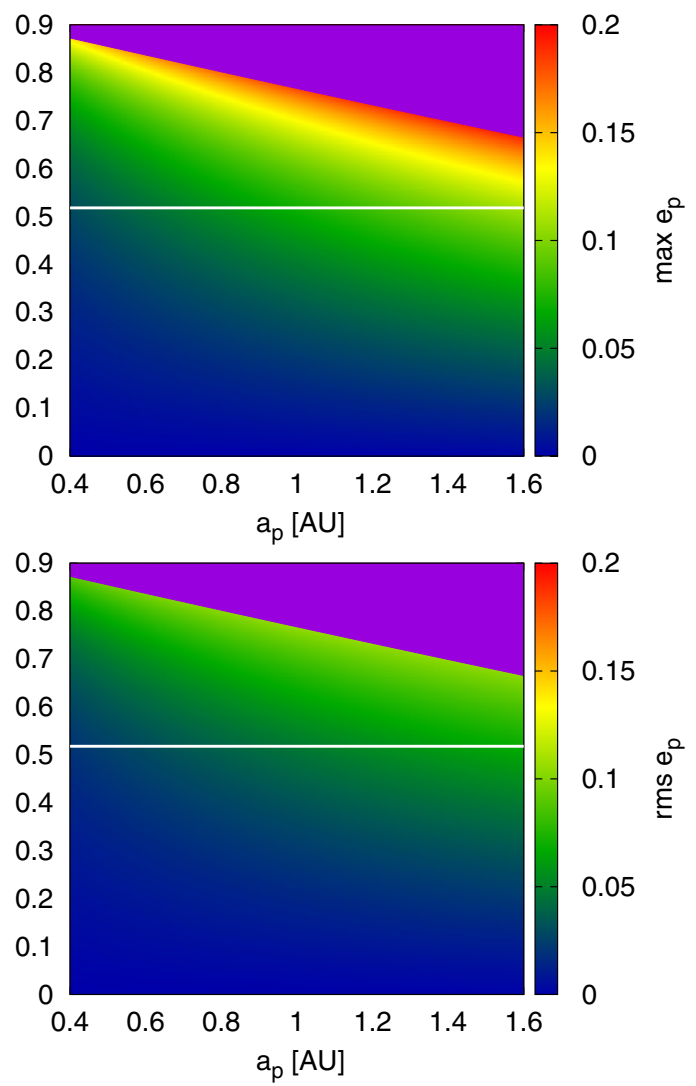

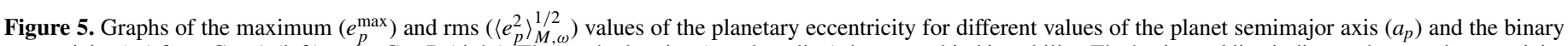

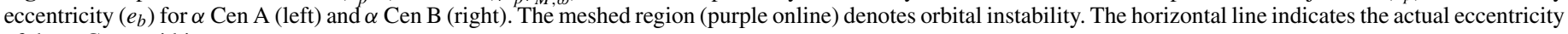
of the $\alpha$ Centauri binary.

(A color version of this figure is available in the online journal.)

in stellar luminosities, the HZs around $\alpha$ Cen A are larger and farther away from the host star compared to $\alpha$ Cen B. Since the binary's mass ratio is close to 0.45 , the gravitational influence of $\alpha$ Cen B is more pronounced on the PHZ of $\alpha$ Cen A. This is a consequence of the larger injected planetary eccentricities $\left(e_{p}\right)$ as can be seen from the top row of Figure 5. The relatively larger gravitational influence of $\alpha$ Cen B onto the HZ of $\alpha$ Cen $\mathrm{A}$ is also mirrored in the fact that the region of dynamical instability (meshed, purple online) reaches toward lower binary eccentricities. The change in the range and configuration of HZs with the change in planetary semimajor axis and eccentricity of the binary is pronounced. A clear shrinking trend for PHZ and EHZ can be observed for high values of the binary's eccentricity. While as shown by Eggl et al. (2012), the AHZ in general expands slightly when the eccentricity is enhanced, Figures 3 and 4 show that in the $\alpha$ Centauri system, this HZ depends only weakly on $e_{b}$, making it the closest approximation to the classical HZ as defined by Kasting et al. (1993). Comparing these results with the existing studies on the HZs for $\alpha$ Cen B such as Guedes et al. (2008) and Forgan (2012), one can see that the values of the inner boundaries of the HZs around $\alpha$ Cen $\mathrm{B}$ as given in Figures 3 and 4 coincide well with the previous studies. Forgan (2012) even found a similar shrinking trend with higher planetary eccentricity. Yet, Forgan (2012) did not take the actual coupling between the planet's eccentricity and the binary's orbit into account. The limits for the outer boundaries of HZ in our model are different from the ones in Forgan (2012) since different climatic assumptions were made. In this work, we used insolation limits for atmospheric collapse assuming a maximum greenhouse atmosphere (Kasting et al. 1993) whereas Forgan (2012) focused on emergence from snowball states.

\subsection{Additional Terrestrial Planets in $\alpha$ Centauri's HZs}

While the classification of HZs presented in the previous section is globally applicable to binary star systems, the analytic estimates to calculate their extent (Eggl et al. 2012) are only strictly valid for three-body systems, e.g., the binary star and a planet. Additional perturbers will influence the shape and size of the HZs. It is thus necessary to investigate which effect the already discovered planet around $\alpha$ Cen B would have on an additional terrestrial planet in $\alpha$ Cen B's HZ.

If the mutual perturbations were large, the $\mathrm{HZ}$ boundaries given in Table 3 would have to be adapted, but $\alpha$ Cen Bb's orbital evolution could also contain clues on the presence-or the absence - of an additional planet. Should the interaction between the inner planet and an additional terrestrial body in the HZ be small, then the HZ boundaries would hold. However, a detection of the habitable planet via its influence on $\alpha$ Cen Bb's orbit would become difficult.

In Figure 6, results of numerical investigations on the coupled orbital evolution of an additional terrestrial planet and $\alpha \mathrm{Cen} \mathrm{Bb}$ are presented. The top row of Figure 6 shows the eccentricity evolution of $\alpha \mathrm{Cen} \mathrm{Bb}$ altered by an additional Earth-like planet at the inner and outer edge of $\alpha$ Cen B's AHZ. The corresponding 

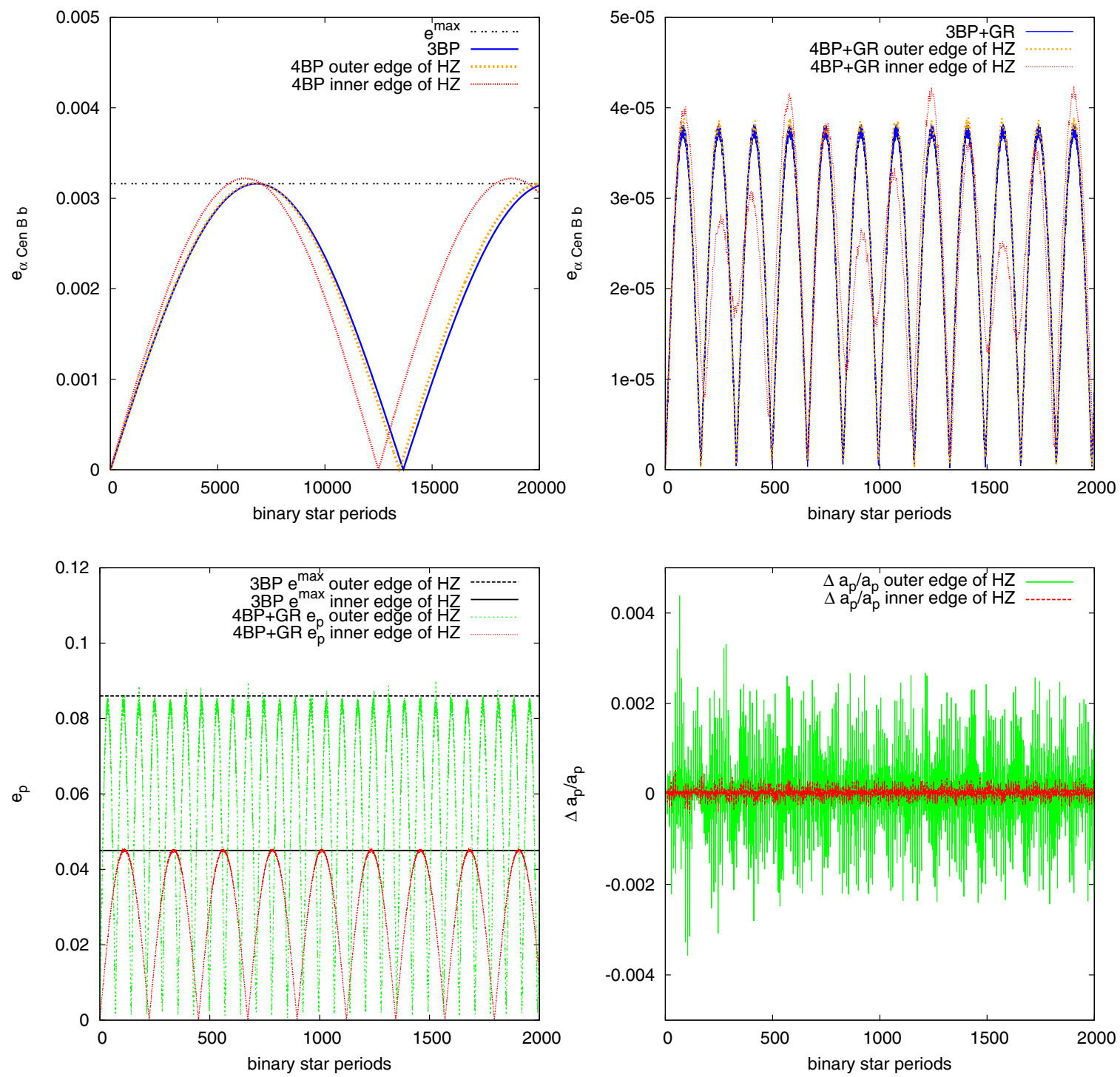

Figure 6. Additional terrestrial planet in $\alpha$ Cen B's $\mathrm{HZ}$ affects the orbit of $\alpha$ Cen $\mathrm{Bb}$ (top row) and vice versa (bottom row). In the top left panel, the numerically computed evolution of the eccentricity of $\alpha \mathrm{Cen} \mathrm{Bb}$ in the Newtonian three-body problem (3BP) consisting of the binary $\alpha$ Cen $\mathrm{AB}$ and the planet $\alpha \mathrm{Cen} \mathrm{Bb}$ is compared with different four-body problem scenarios (4BP). In one scenario, an additional Earth-sized body orbits $\alpha$ Cen B at the inner edge of its AHZ (see Table 3). In the other scenario, the terrestrial planet is assumed to be at the outer edge of $\alpha$ Cen B's AHZ. The analytic estimate for the maximum eccentricity $(e$ max $)$ in the 3BP is presented as well (horizontal line). The top right panel shows the exact same setup, only with general relativity (GR) taken into account. The orbit of $\alpha$ Cen $\mathrm{Bb}$ becomes practically circular. While the influence of an additional planet at the outer edge of the $\mathrm{HZ}$ is barely noticeable in the eccentricity evolution of $\alpha \mathrm{Cen} \mathrm{Bb}$, a planet at the inner edge of the AHZ would cause distinct features. In contrast, neither GR nor $\alpha$ Cen Bb will influence the eccentricity evolution of planets $\left(e_{p}\right)$ in the $\mathrm{HZ}$ significantly as is shown in the bottom left panel. Also, the semimajor axes evolution of additional planets in the HZ is negligible (bottom right panel). Here, $\Delta a_{p} / a_{p}$ denotes the normalized difference between the 4BP+GR and the 3BP semimajor axis evolution of planets at the inner and outer edges of $\alpha$ Cen $\mathrm{B}$ 's AHZ, respectively.

(A color version of this figure is available in the online journal.)

reference curve (dashed, blue online) represents $\alpha$ Cen Bb's eccentricity influenced only by the binary $\alpha$ Cen AB. The top left panel of Figure 6 shows the results in Newtonian three (3BP) and four (4BP) body problems. The top right panel depicts similar analysis with $\mathrm{GR}^{7}$ included. The difference between the two approaches is quite pronounced, as GR clearly prevents the secular rise in Cen Bb's eccentricity predicted in the classical setup (Blaes et al. 2002; Fabrycky \& Tremaine 2007). Thus, the orbit of $\alpha \mathrm{Cen} \mathrm{Bb}$ stays circular, even when tidal forces are neglected. The variations in semimajor axis $(\Delta a)$ for $\alpha$ Cen $\mathrm{Bb}$ are not shown, because they remain below $10^{-8} \mathrm{AU}$ for all cases.

\footnotetext{
7 GR was introduced by numerically solving the Einstein Infeld Hoffman equations (Einstein et al. 1938) for the respective systems.
}

A possible method to search for additional companions is to measure variations in $\alpha$ Cen Bb's orbital period. Yet, the small $\Delta a$ values make this approach difficult, since $\Delta P_{p} \propto P_{p}^{1 / 3} \Delta a$. Disentangling the effects of GR and perturbations due to other habitable planets on $\alpha$ Cen Bb's period would require precisions several orders of magnitude greater than currently available. The top right panel in Figure 6 shows that the perturbations an additional planet at the inner edge of $\alpha$ Cen B's AHZ causes in $\alpha$ Cen Bb's eccentricity are, in principle, distinguishable from the nominal signal. Unfortunately, it is also clear from this graph that neither the required precision nor the observational timescales necessary to identify the presence of an additional Earth-sized companion via observations of $\alpha$ Cen Bb's eccentricity seem obtainable in the near future. For habitable planets at the outer edge of $\alpha$ Cen B's AHZ the chances for indirect detection 
seem even worse, as their influence on $\alpha$ Cen Bb's orbit is negligible.

In order to confirm that the interaction between $\alpha$ Cen $\mathrm{Bb}$ and Earth-like planets in the HZ is small, as well as to further study the influence of the GR on the dynamics of the system, we examined the orbital evolution of a fictitious habitable planet in that region. The results are shown in the bottom row of Figure 6. The left panel depicts the eccentricity evolution of additional terrestrial planets positioned at the inner and outer edges of $\alpha$ Cen B's AHZ. The secular variations in the eccentricity (bottom left panel) and semimajor axis (bottom right panel) of the habitable planet were computed numerically, taking the influence of the binary $\alpha \mathrm{Cen} \mathrm{AB}$, the planet $\alpha \mathrm{Cen} \mathrm{Bb}$, as well as GR into account. When comparing the analytic estimates of $e^{\max }$ with the evolution of the habitable planet's eccentricity in the full system, it is evident that neither GR nor $\alpha$ Cen Bb alter the results for planets in $\alpha$ Cen B's HZ significantly. Also, the deviation in the habitable planet's semimajor axis due to GR and $\alpha \operatorname{Cen~} \mathrm{Bb}\left(\Delta a_{p}\right)$ remains below $0.1 \%$ and $0.5 \%$ for planets at the inner and outer edge of $\alpha$ Cen B's AHZ, respectively.

We conclude that the interaction between additional terrestrial planets in $\alpha$ Cen B's HZ and $\alpha$ Cen Bb is indeed small. Thus, our estimates for the HZs of the $\alpha$ Centauri system remain valid. The existence of additional terrestrial planets on the other hand cannot be determined easily from observing the orbital evolution of $\alpha$ Cen Bb.

The presented results are, strictly speaking, only valid for a coplanar configuration, i.e., the binary and both planets are in the same orbital plane. Mutually inclined configurations can exhibit much more involved dynamics such as Kozai resonant behavior (see, e.g., Correia et al. 2011). A detailed study of such effects lies beyond the scope of this work. Nevertheless, the arguments presented in this section suggest that the search for an additional coplanar planet in the $\mathrm{HZ}$ around $\alpha$ Cen B will most likely have to be performed without relying on observations of $\alpha \mathrm{Cen} \mathrm{Bb}$. We will therefore investigate whether habitable planets can actually be detected independently in Sun-like binary star configurations using current observational facilities.

\subsection{Detectability through Radial Velocity and Astrometry}

We apply our methodology, as derived in Sections 2 and 3, to a fictitious terrestrial planet in the $\mathrm{HZ}$ of binary systems similar to $\alpha$ Centauri AB but with a broadened range of binary eccentricities. In addition to the habitability maps discussed in Section 5.2, Figures 3 and 4 show the results regarding the peak and rms strength of the RV and astrometric signals. Here, the aim is to illustrate how the different types of HZs presented in Section 5.2, as well as the maximum and rms signal strengths defined in Section 2 vary with the binary's eccentricity $\left(e_{b}\right)$ and planetary semimajor axis $\left(a_{p}\right)$. The left column of Figure 3 shows maximum (top) and rms (bottom) values of the signal strengths for the more massive binary component, in this case similar to $\alpha$ Cen A. Results for the less massive component akin to $\alpha$ Cen $\mathrm{B}$ are shown in the right column.

The dashed vertical lines in the top rows of Figures 3 and 4 represent the sections of the parameter space with similar $V_{r}^{\text {circ }}$ and $\rho^{\text {circ }}$ values, respectively. Since $V_{r}^{\text {circ }}$ and $\rho^{\text {circ }}$ are independent of the planetary (and consequently the binary's) eccentricity, the different values of these quantities vary linearly with the planet's semimajor axis. In contrast, $V_{r}^{\max }$ and $\rho^{\max }$, represented by the solid contour lines, depend on the maximum eccentricity of the planet $\left(e_{p}^{\max }\right)$ and therefore change with the binary's eccentricity $\left(e_{b}\right)$. Since for circular binary configurations only small eccentricities are induced into the planet's orbit, $V_{r}^{\max }$ and $V_{r}^{\text {circ }}$ almost coincide. The same holds true for $\rho^{\max }$ and $\rho^{\text {circ }}$ in this case. Yet, $V_{r}^{\max }$ and $\rho^{\max }$ grow with the binary's eccentricity. The corresponding contour lines indicate that for high binary eccentricities even small planetary semimajor axes can produce similar AM peak signal strengths. Similarly, planets with larger distances to their host stars can still cause similar RV amplitudes if the binary's eccentricity is sufficiently large. If a fixed detection limit is set, e.g., $V_{r}=9.5 \mathrm{~m} / \mathrm{s}$, planets with semimajor axes up to $1.5 \mathrm{AU}$ could still be found around stars similar to $\alpha$ Cen A, assuming a binary eccentricity of $e_{b}=0.7$. To produce a similarly high RV amplitude, a circular planet has to orbit its host star at roughly 0.8 AU (Figure 3). In other words, high binary eccentricities lead to excited planetary eccentricities which in turn increase the peak signal strengths suggesting that binary-planet interactions can actually improve the chances for detecting terrestrial planets. Naturally, if the planet's eccentricity happens to be close to zero at the time of observation, this advantage is nullified.

The bottom row of Figures 3 and 4 show the same setup with rms signal strengths $\left\langle\left\langle V_{r}\right\rangle\right\rangle_{M, \omega}$ and $\langle\langle\rho\rangle\rangle_{M, \omega}$, respectively. While $\left\langle\left\langle V_{r}\right\rangle\right\rangle_{M, \omega}$ is independent of the binary's eccentricity, it is evident from Equation (17) that $\langle\langle\rho\rangle\rangle_{M, \omega}$ depends weakly on $e_{b}$ since $\left\langle e_{p}^{2}\right\rangle^{1 / 2} \simeq 0.1$ for the cases considered and therefore $\left\langle e_{p}^{2}\right\rangle \ll 1$ (see Figure 5, bottom). The slight curvature of the contour lines representing the rms signal in Figure 4 indicates this behavior. A summary of RV and AM signal strengths for an Earth-like planet at the boundaries of $\alpha$ Centauri's HZ is presented in Table 3.

We illustrated in this section that the dynamical interactions between a terrestrial planet and the secondary star can produce large peak amplitudes which may enhance the detectability of the planet with the RV and AM methods considerably. The rms values of the planet's AM and RV signals, on the other hand, remain almost unaffected by the gravitational influence of the secondary star.

\subsection{Transit Photometry}

To assess the detectability of a terrestrial planet in the $\mathrm{HZ}$ of $\alpha$ Centauri AB (and similar binaries) through TP, we calculated the relative transit depths that an Earth-like planet would produce during its transit. If such a system hosted a transiting terrestrial planet, TD values would range around $55 \mathrm{ppm}$ for $\alpha$ Cen A, and $115 \mathrm{ppm}$ for $\alpha$ Cen B. Such transit depths are detectable by NASA's Kepler telescope for instance-stellar and instrumental sources included-as the spacecraft's median noise level amounts to $\approx 29 \mathrm{ppm}$ (Gilliland et al. 2011). Therefore, Earth-like planets could in theory be found around $\alpha$ Centauri stars. However, Kepler was not designed to observe stars with apparent magnitudes between 0 and 3 such as those of $\alpha$ Centauri. The Transiting Exoplanets Survey Satellite mission, for instance, will aim for TP of brighter stars (Ricker et al. 2010). Nevertheless, the example of Kepler suggests that the detection of transiting habitable planets in S-type systems would be possible using current technology. In fact, very much similar to the cases discussed in the previous section, the orbit forcing that an Earth-like planet experiences in a binary star system may enhance its possibility of detection via TP (Kane \& von Braun 2008; Kane et al. 2012; Borkovits et al. 2003; Schneider 1994; also see Figure 7). Assuming $\alpha$ Centauri was a transiting system, ${ }^{8}$ a comparison of the transit

\footnotetext{
$8 i_{b}=90^{\circ} \pm \theta_{\text {planet }} / 2$ (Borucki \& Summers 1984).
} 

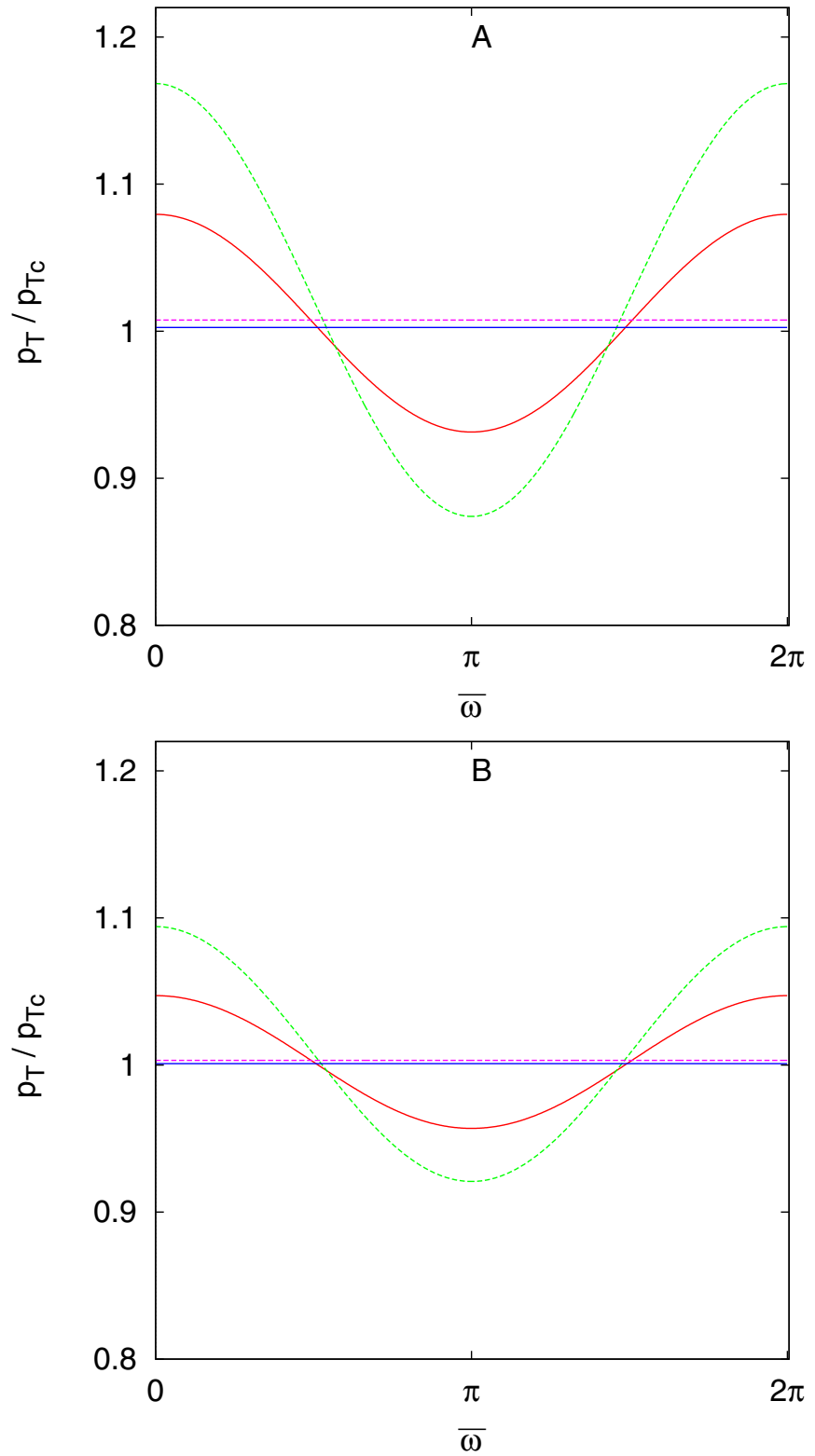

Figure 7. Graphs of the ratio of transit probabilities $\left(p_{T} / p_{T c}\right)$ with $p_{T} \equiv$ $\left.p_{T}\right|_{e=e^{\max }}$ and $\left.p_{T c} \equiv p_{T}\right|_{e=0}$, in a binary similar to the $\alpha$ Centauri system. The graphs show the transit probabilities in terms of the planet's argument of pericenter $(\bar{\omega})$, as measured from the line of sight for component A (top) and component B (bottom). The curved full lines correspond to the planet starting at the inner border of $\mathrm{AHZ}$ and dashed-curved line represents planets that started at the outer edge, for each star (see Table 3). The full and dashed straight lines are the corresponding ratios of the averaged transit probabilities $\left(\left\langle p_{T}\right\rangle /\left\langle p_{T_{c}}\right\rangle\right)$ evaluated using Equation (21). Compared to the transit probability ratios $p_{T} / p_{T c}$, the ratio of averages $\left\langle p_{T}\right\rangle /\left\langle p_{T c}\right\rangle$ shows only a weak dependence on the planet's initial position in the $\mathrm{HZ}$.

(A color version of this figure is available in the online journal.)

probabilities of actual planetary orbits to circular orbits shows that an $18 \%$ increase in $p_{T}$ values seems possible for terrestrial planets at the outer edge of $\alpha$ Cen A's AHZ (Figure 7). Given the right orbital configuration, it may be more likely to identify a transiting habitable terrestrial planet around a stellar component of a binary than around a single star assuming similar initial planetary eccentricities.

The increase in transit probability for planets in double star systems is less dramatic when the equations are averaged over all possible configurations of the argument of pericenter as in Equation (21). Averaged transit probabilities are represented by the straight lines in Figure 7. As $\left\langle p_{T}\right\rangle /\left\langle\left. p_{T}\right|_{e=0}\right\rangle>1$, for terrestrial planets' orbits with $e>0$, the chance for transit is in general higher for Earth-like planets in binary stars than for terrestrial planets in circular orbits around single stars.

\section{DISCUSSION}

Comparing the quantitative estimates of RV, AM, and TP signals, TP seems to be the best choice for finding Earth-like planets in the HZs of a coplanar S-type binary configuration with Sun-like components. Even for a system as near as $\alpha$ Centauri, AM peak signals only measure $\mu$ as. Unfortunately, neither ESO's very long baseline interferometry with PRIMA nor ESA's Gaia mission will be able to deliver such precision in the near future (Quirrenbach et al. 2011). Gaia's aim to provide $\mu$ as AM will most likely not be achieved until the end of the mission (Hestroffer et al. 2010). Also, from an astrometric point of view, Earth-like planets would be easier to find around $\alpha$ Cen $\mathrm{A}$ than $\alpha$ Cen $\mathrm{B}$. That is because the $\mathrm{HZ}$ around star $\mathrm{A}$ is more distant from this star. Naturally, the opposite is true for RV detections. Due to the difference in the stellar masses, $\alpha$ Cen B offers a better chance of finding a terrestrial planet there using RV techniques. The recent discovery of an Earth-sized planet around this star supports our results. The observed planetary RV signal was reproduced excellently by our analytic estimates for circular planetary orbits.

Our prediction of RV amplitudes for terrestrial planets around $\alpha$ Cen $\mathrm{B}$ are also in good agreement with those presented by Guedes et al. (2008). The four terrestrial planets used in the RV model by these authors produce almost exactly four times the predicted rms amplitude given in Figure 3. Guedes et al. (2008) claim that Earth-like planets in the $\alpha$ Centauri are detectable even for signal-to-noise ratios of single observations below 0.1 . However, obtaining sufficient data to reconstruct the planetary signal requires a great amount of dedicated observing time (approximately five years in their example). Validating this statement, it took Dumusque et al. (2012) about four years of acquired data to detect $\alpha \mathrm{Cen} \mathrm{Bb}$. The data published by Dumusque et al. (2012) also allow a glimpse on the current performance of the HARPS spectrograph revealing a precision around $50-80 \mathrm{~cm} \mathrm{~s}^{-1}$. Given the fact that the RV signal of a habitable planet around $\alpha$ Cen B would be still half an order of magnitude smaller (Figure 3), considerably more observation time would be required to identify habitable companions. HIRES measurements are currently yielding precisions around $1 \mathrm{~m} \mathrm{~s}^{-1}$. Identifying RV signals of habitable worlds around $\alpha$ Cen B therefore seems even more unlikely when using HIRES. The previous examples show that some development of observational capacities is still necessary to achieve the RV resolution required for discovering habitable planets in the $\alpha$ Centauri system.

The success of NASA's Kepler space telescope in identifying countless Earth-sized planetary candidates (e.g., Borucki 2011) that require follow-up observations might provide the necessary momentum to develop instruments capable of resolving RV signals in the range of $\mathrm{cm} \mathrm{s}^{-1}$. Focusing on less massive binaries would have the advantage of having greatly enhanced RV signals as the HZs will be situated closer to the planet's host stars. How far this might simplify the task of finding habitable worlds will be the topic of further investigations.

In regard to TP, both Kepler and CoRoT telescopes have proven that it is possible to find terrestrial planets around Sunlike stars (e.g., Léger et al. 2009; Borucki et al. 2012). The 
combination of proven technology and the presented argument that the dynamical environment in binary star systems will enhance transit probabilities makes photometry currently the most promising method for finding Earth-like planets in the HZs of S-type binary star systems.

\section{SUMMARY}

In this work, we provided an analytic framework to estimate the detectability of a terrestrial planet using RV, AM, as well as TP in coplanar S-type binary configurations. We have shown that the gravitational interactions between the stars of a binary and a terrestrial planet can improve the chances for the planet's detection. The induced changes in the planet's eccentricity enhance not only RV and AM peak amplitudes, but also the probability to witness a planetary transit. Next to the presented "best case" estimates, we offered rms/averaged expressions which are deemed to be more suited to determine the longterm influence of the second star on planetary fingerprints in S-type systems. In contrast to peak amplitudes, the rms of a planet's AM signal is only modified slightly by the additional gravitational interaction with the second star. A similar behavior can be seen in planetary transit probabilities. The rms values of RV signals are altogether independent of the secondary's gravitational influence, assuming that the system is nearly coplanar.

After defining the permanent, extended, and average habitable zones for both stellar components of the $\alpha$ Centauri system, we investigated the possible interaction between the newly discovered $\alpha \mathrm{Cen} \mathrm{Bb}$ and additional terrestrial companions in $\alpha$ Cen B's HZ. Our results suggest that $\alpha \mathrm{Cen} \mathrm{Bb}$ is on an orbit with very low eccentricity which would not be influenced significantly by habitable, terrestrial companions. Conversely, $\alpha$ Cen Bb's presence would also not affect Earth-like planets in the HZ of $\alpha$ Cen B.

We estimated the maximum and rms values of the RV as well as AM signal for a terrestrial planet in the $\alpha$ Centauri HZs. The peak and rms amplitudes of the RV signal ranged between 4 and $12 \mathrm{~cm} \mathrm{~s}^{-1}$. Astrometric signals were estimated to lie between 1 and $5 \mu$ as. Given the current observational facilities, enormous amounts of observing time would be required to achieve such precisions. If the $\alpha$ Centauri was a transiting system, however, a habitable planet could be detectable using current technologies. It seems that the detection of Earth-like planets in circumstellar HZs of binaries with Sun-like components via astrometry and $\mathrm{RV}$ is still somewhat beyond our grasp, leaving photometry to be the only current option in this respect.

S.E. and E.P.-L. acknowledge support from FWF through projects AS11608-N16 (EP-L and SE), P20216-N16 (SE and EP-L), and P22603-N16 (EP-L). S.E. acknowledges support from the University of Vienna's Forschungsstipendium 2012. N.H. acknowledges support from the NASA Astrobiology Institute under Cooperative Agreement NNA09DA77A at the Institute for Astronomy, University of Hawaii, and NASA EXOB grant NNX09AN05G. S.E. and E.P-L. also thank the Institute for Astronomy and NASA Astrobiology Institute at the University of Hawaii-Manoa for their kind hospitality during the course of this project. The authors are thankful to Nikolaos Georgakarakos for his valuable suggestions and to the anonymous referee for constructive comments.
APPENDIX A

\section{EQUATION OF THE CENTER}

The equation of the center providing a direct relation between the true anomaly $f$ and the mean anomaly $M$ is presented up to the sixth order in eccentricity $e$ :

$$
\begin{aligned}
f= & M+\left(2 e-\frac{e^{3}}{4}+\frac{5 e^{5}}{96}\right) \sin M \\
& +\left(\frac{5 e^{2}}{4}-\frac{11 e^{4}}{24}+\frac{17 e^{6}}{192}\right) \sin [2 M] \\
& +\left(\frac{13 e^{3}}{12}-\frac{43 e^{5}}{64}\right) \sin [3 M] \\
& +\left(\frac{103 e^{4}}{96}-\frac{451 e^{6}}{480}\right) \sin [4 M] \\
& +\frac{1097}{960} e^{5} \sin [5 M]+\frac{1223}{960} e^{6} \sin [6 M]+O\left(e^{7}\right) .
\end{aligned}
$$

\section{APPENDIX B}

\section{AVERAGING OF $\rho^{2}$}

The averaging integrations over $M$ and $\omega$ in Equations (17) and (18) were carried out as in the following:

$$
\begin{aligned}
& \frac{1}{4 \pi^{2}} \iint_{0}^{2 \pi} \rho^{2}(M, \omega) d M d \omega \\
& =\frac{1}{2 \pi} \int_{0}^{2 \pi} \frac{\mu^{2} a^{2}}{d^{2}}\left\{1+\frac{3\left\langle e^{2}\right\rangle_{M}}{2}\right. \\
& \left.\quad+\left[-\frac{1}{2}+\frac{\left\langle e^{2}\right\rangle_{M}}{4}(5 \cos (2 \omega)-3)\right] \sin ^{2} i\right\} d \omega
\end{aligned}
$$

The integration over $M$ is trivial. Using the partial integration technique to integrate over $\omega$, we obtain

$$
\begin{aligned}
& \frac{1}{2 \pi} \int_{0}^{2 \pi} \frac{5}{4}\left\langle e^{2}\right\rangle_{M} \cos (2 \omega) d \omega \\
& =\frac{5}{4}\left[\left.\left\langle e^{2}\right\rangle_{M, \omega} \cos (2 \omega)\right|_{0} ^{2 \pi}+2\left\langle e^{2}\right\rangle_{M, \omega} \int_{0}^{2 \pi} \sin (2 \omega) d \omega\right]=0 .
\end{aligned}
$$

Here we have used the fact that $\left\langle e^{2}\right\rangle_{M, \omega}=(1 / 2 \pi) \int_{0}^{2 \pi}\left\langle e^{2}\right\rangle_{M} d \omega$ does no longer depend on $\omega$. From the definition of averaging given by Equation (6), we have

$$
\langle\langle\rho\rangle\rangle_{M, \omega}=\frac{\mu a}{2 d}\left[3+\frac{9}{2}\left\langle e^{2}\right\rangle_{M, \omega}+\left(1+\frac{3}{2}\left\langle e^{2}\right\rangle_{M, \omega}\right) \cos (2 i)\right]^{1 / 2} .
$$

A similar procedure has been applied to derive Equation (8).

\section{REFERENCES}

Beaugé, C., Ferraz-Mello, S., \& Michtchenko, T. A. 2007, in Planetary Masses and Orbital Parameters from Radial Velocity Measurements, ed. R. Dvorak (Wiley-VCH), 1

Blaes, O., Lee, M. H., \& Socrates, A. 2002, ApJ, 578, 775

Borkovits, T., Érdi, B., Forgács-Dajka, E., \& Kovács, T. 2003, A\&A, 398, 1091

Borucki, W. J., Koch, D. G., Basri, G., et al. 2011, ApJ, 736, 19

Borucki, W. J., Koch, D. G., Batalha, N., et al. 2012, ApJ, 745, 120

Borucki, W. J., \& Summers, A. L. 1984, Icar, 58, 121 
Chauvin, G., Beust, H., Lagrange, A.-M., \& Eggenberger, A. 2011, A\&A, 528, A8

Correia, A. C. M., Laskar, J., Farago, F., \& Boué, G. 2011, CeMDA, 111, 105

Doyle, L. R., Carter, J. A., Fabrycky, D. C., et al. 2011, Sci, 333, 1602

Dumusque, X., Pepe, F., Lovis, C., et al. 2012, Natur, 491, 207

Eggl, S., Pilat-Lohinger, E., Georgakarakos, N., Gyergyovits, M., \& Funk, B. 2012, ApJ, 752, 74

Einstein, A., Infeld, L., \& Hoffmann, B. 1938, AnMat, 39, 65

Fabrycky, D., \& Tremaine, S. 2007, ApJ, 669, 1298

Ford, E. B., Fabrycky, D. C., Steffen, J. H., et al. 2012, ApJ, 750, 113

Ford, E. B., Quinn, S. N., \& Veras, D. 2008, ApJ, 678, 1407

Forgan, D. 2012, MNRAS, 422, 1241

Georgakarakos, N. 2002, MNRAS, 337, 559

Georgakarakos, N. 2003, MNRAS, 345, 340

Georgakarakos, N. 2005, MNRAS, 362, 748

Gilliland, R. L., Chaplin, W. J., Dunham, E. W., et al. 2011, ApJS, 197, 6

Guedes, J. M., Rivera, E. J., Davis, E., et al. 2008, ApJ, 679, 1582

Haghighipour, N. 2010, Planets in Binary Star Systems (New York: Springer)

Hestroffer, D., Dell'Oro, A., Cellino, A., \& Tanga, P. 2010, in Dynamics of

Small Solar System Bodies and Exoplanets, ed. J. Souchay \& R. Dvorak (Lecture Notes in Physics, Vol. 790; Berlin: Springer), 251

Holman, M. J., \& Wiegert, P. A. 1999, AJ, 117, 621

Kane, S. R., Horner, J., \& von Braun, K. 2012, ApJ, 757, 105

Kane, S. R., \& von Braun, K. 2008, ApJ, 689, 492

Kasting, J. F., Whitmire, D. P., \& Reynolds, R. T. 1993, Icar, 101, 108

Kervella, P., Thévenin, F., Ségransan, D., et al. 2003, A\&A, 404, 1087
Kiseleva-Eggleton, L., \& Eggleton, P. P. 2001, in ASP Conf. Ser. 229, Evolution of Binary and Multiple Star Systems, ed. P. Podsiadlowski, S. Rappaport, A. R. King, F. D’Antona, \& L. Burderi (San Francisco, CA: ASP), 91

Lee, M. H., \& Peale, S. J. 2003, ApJ, 592, 1201

Léger, A., Rouan, D., Schneider, J., et al. 2009, A\&A, 506, 287

Marchal, C. 1990, The Three-body Problem (Amsterdam: Elsevier)

Ohta, Y., Taruya, A., \& Suto, Y. 2005, ApJ, 622, 1118

Orosz, J. A., Welsh, W. F., Carter, J. A., et al. 2012a, A\&A, 758, 87

Orosz, J. A., Welsh, W. F., Carter, J. A., et al. 2012b, Sci, 337, 1511

Paardekooper, S.-J., \& Leinhardt, Z. M. 2010, MNRAS, 403, L64

Pilat-Lohinger, E., \& Dvorak, R. 2002, CeMDA, 82, 143

Pourbaix, D. 2002, A\&A, 385, 686

Pourbaix, D., Nidever, D., McCarthy, C., et al. 2002, A\&A, 386, 280

Quirrenbach, A., Geisler, R., Henning, T., et al. 2011, European Physical Journal Web of Conferences, 16, 7005

Rabl, G., \& Dvorak, R. 1988, A\&A, 191, 385

Ricker, G. R., Latham, D. W., Vanderspek, R. K., et al. 2010, BAAS, 42, 459

Roell, T., Neuhäuser, R., Seifahrt, A., \& Mugrauer, M. 2012, A\&A, 542, A92

Schneider, J. 1994, P\&SS, 24, 539

Schneider, J., Dedieu, C., Le Sidaner, P., Savalle, R., \& Zolotukhin, I. 2011, A\&A, 532, A79

Thébault, P., Marzari, F., \& Scholl, H. 2009, MNRAS, 393, L21

Torres, G., Andersen, J., \& Giménez, A. 2010, A\&ARv, 18, 67

Underwood, D. R., Jones, B. W., \& Sleep, P. N. 2003, IJAsB, 2, 289

Welsh, W. F., Orosz, J. A., Carter, J. A., et al. 2012, Natur, 481, 475

Williams, D. M., \& Pollard, D. 2002, IJAsB, 1, 61 